\title{
DIRECT AGGLUTINATION TEST WITH URINE SAMPLES FOR THE DIAGNOSIS OF VISCERAL LEISHMANIASIS
}

\author{
MOHAMMAD ZAHIDUL ISLAM, MAKOTO ITOH, RUSELLA MIRZA, IFTIKHAR AHMED, \\ A. R. M. SAIFUDDIN EKRAM, ABDUL HALIM SARDER, S. M. SHAMSUZZAMAN, YOSHIHISA HASHIGUCHI, AND \\ EISAKU KIMURA \\ Department of Parasitology, Aichi Medical University School of Medicine, Nagakute, Japan; Department of Endocrinology and \\ Metabolism, Research Institute of Environmental Medicine, Nagoya University, Nagoya, Japan; Department of Microbiology and \\ Department of Medicine, Rajshahi Medical College, Rajshahi, Bangladesh; Department of Parasitology, Kochi Medical School, \\ Kochi, Japan
}

\begin{abstract}
A new direct agglutination test (DAT) for use with urine samples for the diagnosis of visceral leishmaniasis (VL) has been developed and compared with the conventional DAT with serum samples and our previously reported enzyme-linked immunosorbent assay (ELISA) with urine samples (urine ELISA). The new DAT, in which anti-human IgG was used as enhancing antibody, was tested with urine samples from 75 VL patients and 225 non-VL patients and healthy people. The sensitivity of the new DAT (90.7\%), was almost the same as that of the conventional DAT (91.0\%) and the urine ELISA (93.3\%). The specificity of the new DAT (96.4\%) was nearly identical with that of the urine ELISA $(97.3 \%)$. A urine-based DAT has several advantages over the conventional DAT: sample collection is non-invasive and it can process larger numbers of samples with smaller amounts of antigen.
\end{abstract}

\section{INTRODUCTION}

Visceral leishmaniasis (VL) or kala-azar is a potentially fatal disease caused by an intracellular protozoan parasite of the Leishmania donovani complex. It is caused by L. donovani in the Indian subcontinent and eastern Africa, by L. infantum in the entire Mediterranean area and the Middle East, and by L. chagasi in the Latin America, although the latter two species have been shown to be the same by molecular techniques. ${ }^{1}$ More than 47 countries are currently affected by leishmaniasis, with at least 200 million people at risk and approximately 100,000 new cases annually. ${ }^{2}$ Visceral leishmaniasis accounts for 75,000 deaths annually. ${ }^{3}$

For successful control of the disease, efficient and reliable diagnosis of VL is essential. Demonstration of the causative parasites in aspirates from lymph nodes, bone marrow, and the spleen is the most specific diagnosis, and these aspiration materials resulted in a sensitivity of $56.3 \%, 67.1 \%$, and $93.3 \%$, respectively. ${ }^{4}$ However, the techniques are invasive, painful, and hazardous and need skilled personnel and equipped hospitals. Moreover, VL is occurring in places where health services are poorly developed. Poor socioeconomic conditions are associated with a higher risk of infection. ${ }^{5}$

In VL, high antibody levels are observed prior to detection of parasite specific T cell responses, ${ }^{6}$ and several serologic tests, such as the enzyme-linked immunosorbent assay (ELISA), ${ }^{7-12}$ the direct agglutination test (DAT), ${ }^{13-16}$ and the indirect immunofluorescent antibody test (IFAT), ${ }^{17}$ have provided good diagnosis. Among these techniques, the DAT has been well accepted as a routine serologic test due to its simplicity and high sensitivity and specificity. ${ }^{18-21}$ We recently reported an ELISA for use with urine samples (urine ELISA) for the diagnosis of VL that showed a sensitivity of $95.0 \%$ and a specificity of $95.3 \% .{ }^{22}$ An advantage of the urine ELISA is its ease in collecting samples. Since young children are often vulnerable to VL infection, ${ }^{20,23}$ the use of urine will facilitate field studies. However, the ELISA requires expensive equipment that is often not available at laboratories in developing countries. We therefore attempted to use urine in the DAT. As a first step, serum samples were replaced with urine samples in a conventional DAT system. In the second step, antibody (goat anti-human IgG) was added to the sys- tem to improve its sensitivity. The new DAT with enhancing antibody showed similar sensitivity and specificity when compared with the conventional serum-based DAT.

\section{MATERIALS AND METHODS}

Urine and serum samples. Most samples tested in this study were the same ones used in our previous study. ${ }^{22}$ Some additional samples from VL patients and control groups were included. Seventy-five urine samples from defined VL patients, with an average age of 27.4 years, were collected from different medical college hospitals in Bangladesh. Among the 75 patients, 27 were confirmed parasitologically: LeishmanDonovan bodies were detected in splenic aspirates of 13 patients and in bone marrow aspirates of six patients, and promastigotes were demonstrated in eight patients after inoculation of aspirate materials in Novy, MacNeal, and Nicolle medium. Of the other 48 clinically confirmed patients, 43 were positive in the conventional DAT and five were positive in the aldehyde test. During collection of samples, all patients were in the course of treatment with sodium antimony gluconate with the World Health Organization recommended dose. ${ }^{24}$ A total of 225 non-VL urine samples were used. Fiftynine control samples were taken from apparently healthy individuals with no history of kala-azar from endemic areas in Bangladesh (EHC). Sixty-five samples from Japanese healthy individuals were used as non-endemic controls (NEHC). Fifty-eight malaria samples collected in Solomon Islands, 13 tuberculosis samples from Bangladesh, and 23 cutaneous leishmaniasis (CL) samples from Ecuador, and seven samples from patients with other diseases were also included. The other diseases category included two amebic liver abscess patients, two aplastic anemia patients, one aplastic anemia with nephrotic syndrome patient, one aortic stenosis patient, and one viral fever patient. Immediately after collection, $\mathrm{NaN}_{3}$ was added to each sample at the final concentration of $0.1 \%$ as a preservative, and the samples were transported to Japan at ambient temperature and then kept at $4^{\circ} \mathrm{C}$. Fifty-six serum samples from the same group of VL patients were used for the conventional DAT. 
The participants were first told that these samples would be carried to Japan for research purposes only, and not for the diagnosis of their individual disease. All the samples were then collected with the consent of the participants or their parents if they were minors. This study was reviewed and approved by the Ethics Committee of Aichi Medical University School of Medicine and the Ethical Review Committee of the Bangladesh Medical Research Council.

Conventional DAT with serum samples. The DAT with serum was performed using the commercially available antigen produced by Koninklijk Instituut voor de Tropen (Amsterdam, The Netherlands). The test was performed as described by Harith and others. ${ }^{13}$ Briefly, $50 \mu \mathrm{L}$ of two-fold serially diluted serum samples in physiologic saline $(0.15 \mathrm{M} \mathrm{NaCl})$ containing $1 \%$ heat-inactivated fetal bovine serum (FBS) were applied to each well of a $\mathrm{V}$-shaped microtiter plate. The initial serum dilution was 1:200. The DAT antigen $(50 \mu \mathrm{L})$ was then added and after two minutes of gentle shaking on a level surface, the plate was covered with a lid and left overnight at room temperature. The control wells contained physiologic saline plus $1 \%$ heat-inactivated FBS and DAT antigen. The results were judged against a white background. The end point titer was determined in reference to a clear sharpedged blue spot observed in the control wells. Samples with a titer $\geq 1: 3,200$ were considered positive. ${ }^{13}$

DAT with urine samples. Parasite and DAT antigen. Leishmania donovani strain DD8, isolated from a Bangladeshi patient, was used. ${ }^{25}$ Promastigotes were cultured as described previously. ${ }^{22}$ The DAT antigen was prepared following the procedure of Harith and others. ${ }^{13,14}$ Briefly, the log phase promastigotes were harvested, washed five times with cold Locke's solution, $\mathrm{pH} 7.4$, at $4^{\circ} \mathrm{C}$. The pellet was treated with 20 volumes of $0.4 \%$ trypsin at $37^{\circ} \mathrm{C}$ for 45 minutes. The trypsin was removed by centrifugation, the pellet was washed five times with cold Locke's solution, and the treated promastigotes were then fixed overnight with $1 \%$ formaldehyde solution at $4^{\circ} \mathrm{C}$. Following fixation, the promastigotes were washed twice with cold saline $(0.15 \mathrm{M} \mathrm{NaCl}, 0.056 \mathrm{M}$ sodium citrate, $\mathrm{pH}$ 7.4). The promastigotes were then stained with $0.1 \%$ Coomassie brilliant blue R-250 for 90 minutes, washed three times with cold saline, and resuspended at a concentration of $4.5 \times 10^{7}$ promastigotes $/ \mathrm{mL}$ with citrate saline containing $0.4 \%$ formaldehyde solution. They were stored at $4{ }^{\circ} \mathrm{C}$ as DAT antigen. The quality of the antigen was standardized with the commercially available antigen using a positive standard serum sample and other VL serum samples.

DAT without enhancing antibody. A V-shaped 96-well microtiter plate was used. In the first column of the plate, $50 \mu \mathrm{l}$ of dilution buffer (physiologic saline mixed with $2 \%$ volumes of a casein buffer $[1 \%$ casein in $0.05 \mathrm{M}$ Tris- $\mathrm{HCl}, 0.15 \mathrm{M}$ $\mathrm{NaCl}, \mathrm{pH} 7.6]$ )/well was applied. This column served as a negative control. In the first row, $50 \mu \mathrm{L}$ of two-fold serially diluted (with dilution buffer) positive serum, started at a dilution of 1:1,000, was applied to each well a as positive standard to monitor plate-to-plate variations. Fifty microliters of urine sample was applied without concentration or dilution to each of the remaining wells. The DAT antigen $(50 \mu \mathrm{L})$ was then added to each well. After two minutes of gentle shaking on a level surface, the plate was left overnight at room temperature.

The results were judged visually and scored according to the agglutination size. Samples that showed a sharp-edged blue spot identical to the negative control were considered negative (-). Samples showing a full well-sized agglutination were scored plus $3(+++)$. An agglutination $<$ plus 3 but $\geq$ half of plus 3 in diameter was scored plus $2(++)$. A plus 1 agglutination $(+)$ had the size between $(++)$ and $(-)$. Some $(+)$ agglutination showed fine granules around the sharp-edged agglutination.

$D A T$ with enhancing antibody. The type of microtiter plate and positive standard sera were the same as in the previous experiment without antibody, except that physiologic saline instead of dilution buffer was used for the dilution of standard sera and in the negative control wells. Fifty microliters of urine sample was applied as in the previous experiment, to which the same amount of DAT antigen was added. Fifty microliters of goat anti-human IgG (enhancing antibody) (Protos Immunoresearch, San Francisco, CA) was then mixed at various concentrations and times after the addition of antigen. The concentrations and times tested were 1:100, 1:500, $1: 1,000$, and 1:2,000 dilutions with dilution buffer for $0.5,10$, and 20 minutes. The mixture was shaken gently for two minutes and incubated overnight at room temperature.

Judgment of the agglutination results was done as described earlier in this report. Following a series of optimization experiment, it was decided that the enhancing antibody should be diluted 1:500 and mixed immediately after the addition of antigen.

Urine ELISA. The ELISA with urine samples from VL patients and non-VL controls was performed as described elsewhere. ${ }^{22}$ Briefly, flat-bottomed, 96-well microtiter plates (MaxiSorp $^{\mathrm{TM}}$; Nunc, Roskilde, Denmark) were coated with 5 $\mu \mathrm{g} / \mathrm{mL}(100 \mu \mathrm{L} /$ well $)$ of acetone-treated L. donovani antigen and incubated overnight at $4^{\circ} \mathrm{C}$. After blocking with the casein buffer for two hours hr at room temperature, $100 \mu \mathrm{L}$ of urine (1:10 dilution in casein buffer) was added, and the wells were incubated at $37^{\circ} \mathrm{C}$ for $1 \mathrm{hr}$. After four washes with phosphate-buffered saline (PBS), $\mathrm{pH} 7.4$ containing $0.05 \%$ Tween 20 , the plates were incubated with peroxidase-conjugated goat anti-human IgG (Tago, Camarillo, CA) (1:4,000 dilution in casein buffer) at $37^{\circ} \mathrm{C}$ for one hour. The plates were then washed four times with PBS, $0.05 \%$ Tween $20, \mathrm{pH} 7.4$, incubated with substrate (2,2'-azino-bis(3-ethylbenzthiazoline-6sulfonic acid) [ABTS]; Kirkegaard and Perry Laboratories, Gaithersburg, MD) for one hour at room temperature, and the optical density was measured at $415 \mathrm{~nm}$ and $492 \mathrm{~nm}$ as a reference. Each sample was assayed in duplicate.

Antibody levels were expressed as units estimated from a standard curve constructed with serially diluted positive sera. The cut-off point for IgG antibodies to $L$. donovani was calculated as the mean plus three standard deviations of log $(1+$ unit) values of the NEHC. The anti-logarithmic cutoff value (9.0 units) in this study is slightly different from that (7.4 units) determined in our previous study; new batches of antigen and a larger number of NEHC might have caused the difference.

\section{RESULTS}

DAT with urine samples. Urine samples of $57 \mathrm{VL}$ and 137 non-VL patients and healthy people were tested with urinebased DAT without goat anti-human IgG. The assay showed a sensitivity of $68.4 \%$ (39 of 57 VL samples were positive) and a specificity of $94.1 \%$ (129 of 137 non-VL samples were nega- 
TABLE 1

Sensitivity and specificity of a urine-based direct agglutination test without anti-human $\operatorname{IgG} *$

\begin{tabular}{|c|c|c|c|c|c|c|}
\hline \multirow[b]{2}{*}{ Agglutination score } & \multirow{2}{*}{$\frac{\text { Sensitivity } \dagger(\%)}{\mathrm{VL}}$} & \multicolumn{5}{|c|}{ Specificity (\%) } \\
\hline & & EHC & M & ТВ & OD & Total \\
\hline & 12 & 0 & 2 & 0 & 2 & 4 \\
\hline & 14 & 0 & 1 & 0 & 0 & 1 \\
\hline & 13 & 1 & 2 & 0 & 0 & 3 \\
\hline - & 18 & 58 & 53 & 13 & 5 & 129 \\
\hline Total & 39/57 (68.4) & $58 / 59(98.3)$ & 53/58 (91.4) & 13/13 (100) & 5/7 (71.4) & 129/137 (94.1) \\
\hline
\end{tabular}

$* \mathrm{VL}=$ visceral leishmaniasis; $\mathrm{EHC}=$ healthy controls from endemic areas; $\mathrm{M}=$ malaria; $\mathrm{TB}=$ tuberculosis; $\mathrm{OD}=$ other diseases.

$\dagger$ Number of positive/total number of VL samples.

$\ddagger$ Number of negative/total number of non-VL samples in each category.

tive) (Table 1). Among 39 VL-positive samples, 12 (30.8\%) were graded as plus 3, $14(35.9 \%)$ as plus 2, and $13(33.3 \%)$ as plus 1 . The specificity of this assay with EHC, malaria, tuberculosis, and other diseases was $98.3 \%, 91.4 \%, 100 \%$, and $71.4 \%$, respectively. Among the false-positive samples, two malaria and two other diseases (aplastic anemia and aplastic anemia with nephrotic syndrome) were plus 3 , one malaria was plus 2, and two malaria and one EHC were plus 1 .

The addition of enhancing antibody greatly improved the urine-based DAT. With $75 \mathrm{VL}$ and 225 non-VL samples, it showed a sensitivity of $90.7 \%$ ( 68 of $75 \mathrm{VL}$ samples were positive) and a specificity of $96.4 \%$ (217 of 225 non-VL samples were negative) (Table 2). Among $68 \mathrm{VL}$-positive samples $46(67.6 \%)$ were graded as plus 3 , and $11(16.2 \%)$ each as plus 2 or plus 1 . Seven of 75 samples became false negative, of which four samples were collected from parasitologically confirmed patients. The specificity of this assay with EHC, NEHC, malaria, tuberculosis, CL, and other diseases was $96.6 \%, 100 \%, 100 \%, 92.3 \%, 87.0 \%$, and $71.4 \%$, respectively. Although the specificity did not improve much with or without the antibody, its addition reduced the degree of false reactivity, that is, all false-positive samples showed plus 1 agglutination.

DAT with serum samples. The sensitivity of the conventional DAT was studied with serum samples of $56 \mathrm{VL}$ patients. Due to a lack of samples, the specificity could not be determined. The assay showed a sensitivity of $91.1 \%$ (51 of 56 VL samples were positive).

The urine-based DAT with enhancing antibody was compared with conventional serum-based DAT with 56-paired urine and serum samples from defined VL cases. There was a clear positive correlation between serum and urine DAT results ( $\mathrm{r}=0.6078, P<0.001$, Spearman's correlation coefficient by rank test) (Figure 1). Fifty samples were positive and four samples were negative in both assays (Table 3). Among the four false-negative samples, three were from parasitologically confirmed patients. One parasitologically positive and conventional DAT-positive sample became negative in the urine-based DAT. One parasitologically positive but conventional DAT-negative sample became positive in the urinebased DAT.

Urine ELISA. Urine samples of $75 \mathrm{VL}$ patients and 225 non-VL patients and healthy people were tested with the ELISA using acetone-treated antigen. The test showed a sensitivity of $93.3 \%$ ( 70 of 75 VL samples were positive) and a specificity of $97.3 \%$ (219 of 225 non-VL samples were negative) (Table 4). The specificity of this assay with EHC, NEHC, malaria, tuberculosis, CL, and other diseases was similar to that of the urine-based DAT with enhancing antibody.

When we compared the urine-based DAT with enhancing antibody with the urine ELISA using 75 VL samples, 68 samples became positive and five samples became negative in both assays (Table 3). Three of the five false-negative samples were collected from parasitologically confirmed patients. Two urine ELISA-positive samples, one of which was parasitologically positive, became negative in the urine-based DAT. The antibody units of these two ELISA positive samples were 35.2 units and 11.9 units.

\section{DISCUSSION}

The phlebotomine sand flies transmit VL to humans. Soon after the insect bite, parasites are found in macrophage phagolysosomes of hosts. Efficient cellular immune responses to these parasites can alleviate clinical symptoms and result in cure. ${ }^{26}$ With insufficient immune responses, however, the pa-

TABLE 2

Sensitivity and specificity of a urine-based direct agglutination test with anti-human $\operatorname{IgG}^{*}$

\begin{tabular}{|c|c|c|c|c|c|c|c|c|}
\hline \multirow[b]{2}{*}{ Agglutination score } & \multirow{2}{*}{$\frac{\text { Sensitivity } \nmid(\%)}{V L}$} & \multicolumn{7}{|c|}{ Specificity } \\
\hline & & EHC & NEHC & M & TB & $\mathrm{CL}$ & OD & Total \\
\hline+++ & 46 & 0 & 0 & 0 & 0 & 0 & 0 & 0 \\
\hline++ & 11 & 0 & 0 & 0 & 0 & 0 & 0 & 0 \\
\hline+ & 11 & 2 & 0 & 0 & 1 & 3 & 2 & 8 \\
\hline- & 7 & 57 & 65 & 58 & 12 & 20 & 5 & 218 \\
\hline Total & 68/75 (90.7) & $57 / 59(96.6)$ & 65/65 (100) & 58/58 (100) & 12/13 (92.3) & 20/23 (87.0) & $5 / 7(71.4)$ & $217 / 225(96.4)$ \\
\hline
\end{tabular}

$* \mathrm{VL}=$ visceral leishmaniasis; $\mathrm{EHC}=$ healthy controls from endemic areas; $\mathrm{NEHC}=$ healthy controls from non-endemic areas; $\mathrm{M}=$ malaria; $\mathrm{TB}=$ tuberculosis; $\mathrm{CL}=$ cutaneous leishmaniasis; OD = other diseases.

$\dagger$ Number positive/total number of VL samples.

$\doteqdot$ Number negative/total number of non-VL samples in each category. 


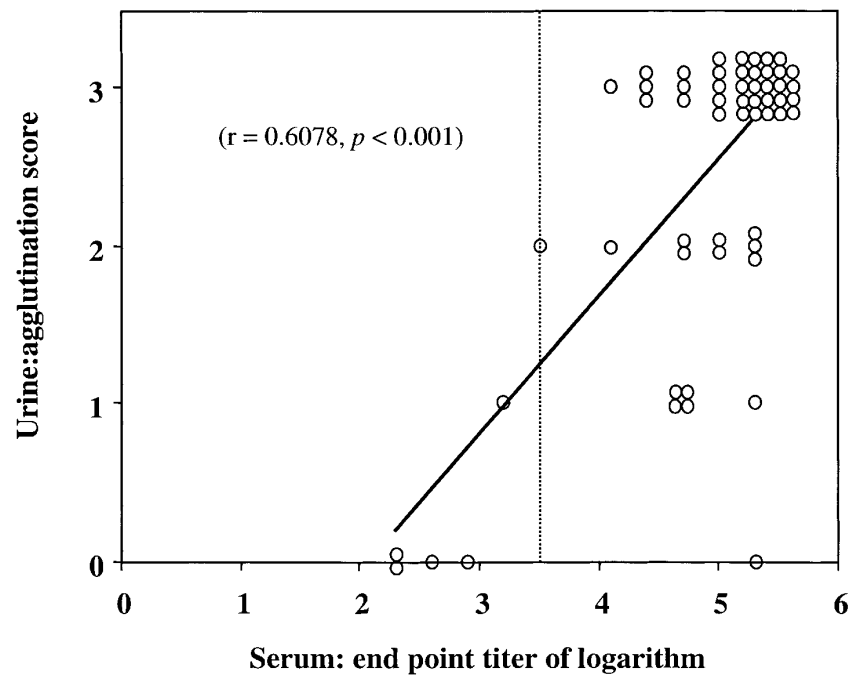

FIGURE 1. Correlation between a urine-based direct agglutination test (DAT) with enhancing antibody and a conventional serum-based DAT. The vertical dotted line is the cut-off level for the serum-based DAT.

tient may die, in many cases, of pancytopenia. The incubation period generally varies from two to six months, but it may have much wider range. Visceral leishmaniasis is targeted for control under the World Health Organization Special Program for Research and Training in Tropical Diseases. The effective control of VL has been hampered due to the lack of suitable tools for early detection of infection and effective chemotherapy. Recently, a long-awaited oral drug, miltefosine, has been shown to be very effective for the treatment of Indian VL. ${ }^{27}$ In the near future, with the advent of reliable early diagnosis, patients could be successfully treated with oral drugs on an outpatient basis.

In this report, we described a urine-based DAT for the diagnosis of VL cases. A urine-based DAT without enhancing antibody showed a sensitivity of $68.4 \%$ and a specificity of $94.1 \%$. We succeeded in improving the sensitivity and specificity by adding goat anti-human IgG to the DAT system. The DAT with enhancing antibody showed a sensitivity of $90.7 \%$ and a specificity of $96.4 \%$. Addition of the antibody probably strengthened antibody-mediated bindings between antigen particles and facilitated the agglutination.

When the sensitivities of the urine-based DAT with the antibody (90.7\%), the serum-based conventional DAT $(91.0 \%)$, and the urine ELISA $(93.3 \%)$ were compared, there was no difference among three methods $(P>0.8$, by chi-

TABLE 3

Comparison of a urine-based DAT with enhancing antibody with a conventional serum-based DAT (left) and with a urine ELISA (right) with VL patient samples*

\begin{tabular}{crrrrrr}
\hline & \multicolumn{2}{c}{ DAT with urine } & & \multicolumn{2}{c}{ DAT with urine } \\
\cline { 2 - 3 } & + & - & & + & - \\
\hline DAT with serum & & & Urine ELISA & & \\
+ & 50 & 1 & + & 68 & 2 \\
- & 1 & 4 & - & 0 & 5 \\
\hline
\end{tabular}

* DAT $=$ direct agglutination test; ELISA $=$ enzyme-linked immunosorbent assay; $\mathrm{VL}=$ visceral leishmaniasis.
TABLE 4

Sensitivity and specificity of an enzyme-linked immunosorbent assay with urine samples*

\begin{tabular}{|c|c|c|c|c|c|c|c|}
\hline Sensitivitył (\%) & & & & pecificity: & (\%) & & \\
\hline VL & EHC & NEHC & M & TB & $\mathrm{CL}$ & $\mathrm{OD}$ & Total \\
\hline $\begin{array}{l}70 / 75 \\
(93.3)\end{array}$ & $\begin{array}{l}58 / 59 \\
(98.3)\end{array}$ & $\begin{array}{l}65 / 65 \\
(100)\end{array}$ & $\begin{array}{l}58 / 58 \\
(100)\end{array}$ & $\begin{array}{l}13 / 13 \\
(100)\end{array}$ & $\begin{array}{l}20 / 23 \\
(87.0)\end{array}$ & $\begin{array}{c}5 / 7 \\
(71.4)\end{array}$ & $\begin{array}{c}219 / 225 \\
(97.3)\end{array}$ \\
\hline
\end{tabular}

$* \mathrm{VL}=$ visceral leishmaniasis; $\mathrm{EHC}=$ healthy controls from endemic areas; $\mathrm{NEHC}=$ healthy controls from non-endemic areas; $\mathrm{M}=$ malaria; $\mathrm{TB}=$ tuberculosis; $\mathrm{CL}=$ cutaneous leishmaniasis; OD = other diseases.

$\dagger$ Number of positive/total number of VL samples.

$¥$ Number of negative/total number of non-VL samples in each category.

square test). Also, there was no difference in the specificities between the urine-based DAT with the antibody $(96.4 \%)$ and the urine ELISA $(97.3 \%)(P>0.5$, by chi-square test). All three methods showed relatively low specificity with CL and other diseases. The CL samples might be more cross-reactive than other non-VL categories due to the parasitologic similarity of the causative agents. Two aplastic anemia samples were positive. It seems to be important to study these patients because both aplastic anemia and VL cause pancytopenia.

The conventional serum-based DAT and ELISA have been evaluated in various studies. The DAT showed a sensitivity of $92.0-96.5 \%$ and a specificity of $99.4-100 \% .^{15,21,28}$ In a recent study in Brazil, the recombinant K39-based ELISA and strip test showed a sensitivity of $90 \%$ and $89 \%$ and a specificity of $100 \%$ and $98 \%$, respectively. ${ }^{29}$ These sensitivity and specificity values are consistent with those of our newly developed urine-based DAT.

In the conventional DAT, serial dilution of serum samples is necessary to determine the end point titer, which requires a large volume of antigen for testing a single sample. Seeking simplicity, Harith and others proposed that a single dilution of 1:1,600 be used in a mass screening program. ${ }^{14}$ However Boeleart and others ${ }^{28}$ strongly opposed the use of a single, universal DAT cut-off titer for all purposes. They suggested that a DAT cut-off titer had to be made in the context of variable local conditions. In our urine-based DAT with enhancing antibody, samples were used without serial dilution. We can process a larger number of samples in one plate, which will reduce the amount of antigen, cost, time, and labor/ test. The cost of enhancing antibody is much cheaper than that of the DAT antigen needed for serial dilution. Moreover, the use of urine samples has definite advantages in the field because of non-invasiveness in collecting samples. In the village where many people had experienced blood sampling, urine collection was accepted well or even welcomed. ${ }^{30}$ Another advantage is that this assay system does not require the electrical equipment needed for the ELISA. Thus, this method could be a very useful diagnostic tool for VL especially in the field.

Received July 2, 2003. Accepted for publication September 9, 2003.

Acknowledgments: We thank Dr. Farzana Matin, Dr. Mohammed Mohsin Ali, Dr. A. K. M. Shamsuzzaman Choudhury, Dr. Mohammed Akram Hossain, and Dr. Nllufar Begam for helping in the collection of VL samples, and Dr. Judson. L. Leafasia for providing malaria samples. We also thank Dr. Mohammed Khaled Hossain and Dr. Mirza Mohammed Shamim for transporting some VL samples from Bangladesh to Japan.

Financial support: This study was supported by a Grant-in-Aid for Scientific Research (B) No. 15406018 and a Grant-in-Aid for Scien- 
tific Research (C) No. 12670243 from the Japan Society for the Promotion of Science.

Authors' addresses: Mohammad Zahidul Islam, Makoto Itoh, and Eisaku Kimura, Department of Parasitology, Aichi Medical University School of Medicine, Nagakute, Aichi-ken 480-1195, Japan, Telephone: 81-52-264-4811, Fax: 81-561-63-3645 E-mails: zahid@aichimed-u.ac.jp, macitoh@aichi-med-u.ac.jp, and kimura@aichi-med-u. ac.jp. Rusella Mirza, Department of Endocrinology and Metabolism, Research Institute of Environmental Medicine, Nagoya University, Furo-cho, chikusa-ku, Nagoya 464-8601, Japan, Telephone: 81-52789-3867, Fax: 81-52-789-3891, E-mail: rusella17@yahoo.com. Iftikhar Ahmed, Department of Microbiology, Rajshahi Medical College, Rajshahi, Bangladesh, Telephone: 88-721-776001-9, Fax: 88-52721-772174, E-mail: ia@librabd.net. A. R. M. Saifuddin Ekram and Abdul Halim Sarder, Department of Medicine, Rajshahi Medical College, Rajshahi, Bangladesh, Telephone: 88-721-776001-9, Fax: +88-52-721-772174, E-mail: rmc@mail.librabd.net. S. M. Shamsuzzaman and Yoshihisa Hashiguchi, Department of Parasitology, Kochi Medical School, Nankoku City, Kochi 783-8505, Japan, Telephone: 81-88-880-2415, Fax: 81-88-880-2415, E-mail: hasiguti@med.kochi-ms. ac.jp.

Reprint requests: Mohammad Zahidul Islam, Department of Parasitology, Aichi Medical University School of Medicine, Nagakute, Aichi-ken 480-1195, Japan.

\section{REFERENCES}

1. Mauricio IL, Stotthard JR, Miles MA, 2000. The strange case of Leishmania chagasi. Parasitol Today 16: 188-189.

2. Ashford R W, Desjeux P, de Raadt P, 1992. Estimation of population at risk of infection and number of cases of leishmaniasis. Parasitol Today 8: 104-105.

3. Wijeyarante PM, Arsenault LK, Murphy CJ, 1994. Endemic disease and development: the leishmaniasis. Acta Trop 56: 349364.

4. Zijlstra EE, Ali MS, el-Hassan AM, el-Toum IA, Satti M, Ghalib HW, Kager PA, 1992. Kala-azar: a comparative study of parasitological methods and the direct agglutination test in diagnosis. Trans R Soc Trop Med Hyg 86: 505-507.

5. Cerf BJ, Jones TC, Badaro R, Sampaio D, Teixeira R, Jr Johnson WD, 1987. Malnutrition as a risk factor for severe visceral leishmaniasis. J Infect Dis 156: 1030-1033.

6. Ghose AC, Haldar JP, Pal SC, Mishra BP, Mishra KK, 1980. Serological investigations on Indian kala-azar. Clin Exp Immunol 40: 318-326.

7. Amin ERME, Wright PA, Kager PA, Laarman JJ, Pondman KW, 1985. ELISA using intact promastigotes for immunodiagnosis of kala-azar. Trans $R$ Soc Trop Med Hyg 79: 344-350.

8. Fargeas C, Hommel M, Maingon R, Dourado C, Monsigny M, Mayer R, 1996. Synthetic peptide-based enzyme-linked immunosorbent assay for serodiagnosis of visceral leishmaniasis. J Clin Microbiol 34: 241-248.

9. Jaffe CL, McMahon-Pratt D, 1987. Serodiagnostic assay for visceral leishmaniasis employing monoclonal antibodies. Trans $R$ Soc Trop Med Hyg 81: 587-594.

10. Kaul P, Malla N, Kaur S, Mahajan RC, Ganguly NK, 2000. Evaluation of a $200-\mathrm{kDa}$ amastigote-specific antigen of $L$. donovani by enzyme-linked immunosorbent assay (ELISA) for the diagnosis of visceral leishmaniasis. Trans $R$ Soc Trop Med Hyg 94: $173-175$.

11. Raj VS, Ghosh A, Dole VS, Madhubala R, Myler PJ, Stuart KD, 1999. Serodiagnosis of leishmaniasis with recombinant ORFF antigen. Am J Trop Med Hyg 61: 482-487.

12. Zijlstra EE, Daifalla NS, Kager PA, Khalil EAG, Hassan AME, S. G. Reed, H. W. Ghalib. 1998. rK39 enzyme-linked immunosorbent assay for diagnosis of Leishmania donovani infection. Clin Diagn Lab Immunol 5: 717-720.

13. Harith AE, Kolk AHJ, Leeuwenburg J, Muigai R, Kiugu S, Kiugu S, Laarman JJ, 1986. A simple and economical direct agglutination test for serodiagnosis and sero-epidemiological studies of visceral leishmaniasis. Trans $R$ Soc Trop Med Hyg 80: $583-587$.

14. Harith AE, Kolk AHJ, Leeuwenburg J, Muigai R, Huigen E, Jelsma T, Kager PA, 1988. Improvement of a direct agglutination test for field studies of visceral leishmaniasis. J Clin Microbiol 26: 1321-1325.

15. Meredith SEO, Kroon NCM, Sondorp E, Seaman J, Goris MGA, van Ingen $\mathrm{CW}$, Oosting $\mathrm{H}$, Schoone GJ, Terpstra WJ, Oskam L, 1995. Leish-KIT, a stable direct agglutination test based on freeze-dried antigen for serodiagnosis of visceral leishmaniasis. J Clin Microbiol 33: 1742-1745.

16. Walton BC, Brooks VM, Arojone I, 1972. Serodiagnosis of American leishmaniasis by indirect fluorescent antibody test. Am J Trop Med Hyg 21: 296-299.

17. Mbati PA, Githure JI, Kagai JM, Kirigi G, Kibati F, Wasunna K, Koech D. K, 1999. Evaluation of a standardized direct agglutination test (DAT) for the diagnosis of visceral leishmaniasis in kenya. Ann Trop Med Parasitol 93: 703-710.

18. Addy M, Som DK, Das C, Bhattacharya S, Bowmik T, Rakshit P, Patra P, Nandy A Choudhury, AB, 1989. Evaluation of direct agglutination test (DAT) in the diagnosis and screening of kala-azar. Indian Med Gaz 123: 184-187.

19. Chowdhury MS, al Masum A, al Karim E, Semiao-Santos S, Rahman KM, Ar-Rashid H, el Harith A, 1993. Applicability of direct agglutination test (DAT) at a rural health setting in Bangladesh and feasibility of local antigen production. Arch Inst Pasteur Tunis 70: 333-344.

20. Chowdhury S, Haque F, Masum AA, Harith A, Karim E, 1991. Positive response to sodium antimony gluconate adminstration in visceral leishmaniasis seropositive patients. Am J Trop Med Hyg 44: 390-393.

21. Singla N, Singh GS, Sundar S, Vinayak VK, 1993. Evaluation of direct agglutination test as an immunodiagnostic tool for kalaazar in India. Trans R Soc Trop Med Hyg 87: 276-278.

22. Islam MZ, Itoh $M$, Shamsuzzaman SM, Mirza R, Matin F, Ahmed I, Choudhury AKMS, Hossain MA, Qiu XG, Begam N, Furuya M, Leafasia JL, Hashiguchi Y, Kimura E, 2002. Diagnosis of visceral leishmaniasis by ELISA using urine samples. Clin Diagn Lab Immunol 9: 789-794.

23. Badaro R, Jones TC, Lorenco R, Cerf BJ, Sampio D, Carvalho EM, Rocha H, Teixeira R, Jr Johnson WD, 1986. A prospective study of visceral leishmaniasis in an endemic area of Brazil. J Infect Dis 154: 639-649.

24. World Health Organization, 1984. Leishmaniasis. World Health Organ Tech Rep Ser 701.

25. Shamsuzzaman SM, Furuya M, Choudhury AKMS, Korenaga M, Hashiguchi Y, 2000. Characterisation of Bangladeshi Leishmania isolated from kala-azar patients by isoenzyme electrophoresis. Parasitol Int 49: 139-145.

26. Badaro R, Jones TC, Carvalho EM, Sampaio D, Reed SG, Barral A, Teixeira R, Johnson WD Jr, 1986. New perspectives on a subclinical form of visceral leishmaniasis. J Infect Dis 154 : 1003-1011.

27. Jha TK, Sundar S, Thakur CP, Backmann P, Karbwang J, Fischer C, Vos A, Berman J, 1999. Miltefosine, an oral agent, for the treatment of Indian visceral leishmaniasis. $N$ Engl J Med 341: 1795-1800.

28. Boelaert M, El Safi S, Jacquet D, de Muynck A, van der Stuyft P, Le Ray D, 1999. Operational validation of the direct agglutination test for diagnosis of visceral leishmaniasis. Am J Trop Med Hyg 60: 129-134.

29. Carvalho SFG, Lemos EM, Core R, Dietze R, 2003. Performance of recombinant $\mathrm{K} 39$ antigen in the diagnosis of Brazilian visceral leishmaniasis. Am J Trop Med Hyg 68: 321-324.

30. Weerasooriya MV, Itoh M, Islam MZ, Qiu XG, Fujimaki Y, Kimura E, 2003. Prevalence and levels of filaria-specific urinary IgG4 among children less than five years of age and the association of the antibody positivity between the children and their mothers. Am J Trop Med Hyg 68: 465-468. 